\begin{tabular}{|c|c|}
\hline Title & Threshold-variation-enhanced adaptability of response in a nanowire field-effect transistor network \\
\hline Author(s) & Kasai, Seiya; Miura, Kensuke; Shiratori, Y uta \\
\hline Citation & $\begin{array}{l}\text { A pplied Phy sics Letters, 96(19), } 194102 \\
\text { https://doi.org/10.1063/1.3428784 }\end{array}$ \\
\hline Issue Date & $2010-05-10$ \\
\hline Doc URL & http:/hdl.handle.net/2115/43100 \\
\hline Rights & $\begin{array}{l}\text { Copyright } 2010 \text { A merican Institute of Phy sics. This article may be downloaded for personal use only. A ny other use } \\
\text { requires prior permission of the author and the A merican Institute of Physics. The following article appeared in A ppl. } \\
\text { Phys. Lett. } 96,194102 \text { (2010) and may be found at https://dx.doi.org } / 10.1063 / 1.3428784\end{array}$ \\
\hline Type & article \\
\hline File Information & APL96-19_194102.pdf \\
\hline
\end{tabular}

Instructions for use 


\title{
Threshold-variation-enhanced adaptability of response in a nanowire field-effect transistor network
}

\author{
Seiya Kasai, ${ }^{1}{ }^{1,2, a)}$ Kensuke Miura, ${ }^{1}$ and Yuta Shiratori ${ }^{1}$ \\ ${ }^{1}$ Graduate School of Information Science and Technology, and Research Center for Integrated Quantum \\ Electronics, Hokkaido University, N14, W9, Sapporo 060-0814, Japan \\ ${ }^{2}$ PRESTO, Japan Science and Technology Agency, 4-1-8, Honcho, Kawaguchi-shi, Saitama 332-0012, Japan
}

(Received 3 April 2010; accepted 21 April 2010; published online 12 May 2010)

\begin{abstract}
Stochastic resonance in a summing network with varied thresholds was investigated using GaAs-based etched nanowire field-effect transistors having different threshold voltages. The network's response adapted to input offset fluctuations in the range of the threshold voltage variation and the network could detect a weak signal without any adjustment of the input offset or the addition of high noise. The observed adaptability resulted from a widened dynamic range of the system due to signal decomposition and reconstruction by multiple thresholds together with the output summation process. (C) 2010 American Institute of Physics. [doi:10.1063/1.3428784]
\end{abstract}

The coexistence of noise and fluctuations created by utilizing stochastic resonance (SR) has recently been an interesting issue in electronics. ${ }^{1-4} \mathrm{SR}$ is a phenomenon in which the response of a system is enhanced by adding noise. ${ }^{5,6}$ It is known to be a key mechanism for precise detection and transmission of weak signals comparable to the thermal energy in biological systems. ${ }^{7}$ To date, many electronic systems that artificially cause SR have been investigated ${ }^{8-12}$ but they have never been turned into practical applications excepting dithering. A major reason is that the SR at an optimal noise never improves the information content of the signal as that before adding noise. However it can improve the information transfer and the signal-to-noise ratio with noise, ${ }^{13,14}$ and this feature is still useful for electronics. We have succeeded in causing SR in a semiconductor nanowire field-effect transistor (FET) and have demonstrated an enhancement of the response of an FET parallel summing network. ${ }^{15}$ At this stage, the positive contribution of noise has been confirmed, but the influence of the physical fluctuation of network units on SR has not been clarified, although Collins et al. ${ }^{16}$ suggested that the noise compensates for the variation in units leading to an optimally enhanced system response. In this Letter, we report on our investigation of the signal response of a summing network of GaAs-based nanowire FETs with threshold voltage variation. The threshold voltages of seven FETs were artificially modified by making devices of different sizes. A parallel summing network was formed using the different FETs and the system's SR response was characterized experimentally.

An FET summing network with varied threshold voltages is schematically shown in Fig. 1(a). The basic mechanism of SR is a noise-assisted state transition in a threshold system. ${ }^{6,13}$ To cause SR, we utilized the nonlinearity of the FET gate threshold characteristic. ${ }^{15}$ The input and output of this system were gate voltage $V_{\mathrm{G}}$ and the sum of the sourcedrain current from each FET $\Sigma I_{\mathrm{DS}}\left(=I_{\text {out }}\right)$, respectively. The FET was operated in the subthreshold region. The FETs were fabricated on nanowires formed by electron beam lithogra-

\footnotetext{
${ }^{\text {a) }}$ Author to whom correspondence should be addressed. Electronic mail: kasai@rciqe.hokudai.ac.jp. Tel.: +81-11-706-6509. FAX: +81-11-7166004.
}

phy and wet chemical etching of a conventional AlGaAs/ GaAs modulation-doped structure with a two-dimensional electron gas. A Schottky wrap gate (WPG) was formed on each nanowire. An example of a fabricated FET is shown in Fig. 1(b). The threshold voltage $V_{\text {th }}$ of the present nanowire FET is known to depend on nanowire width $W$ and WPG gate length $L_{\mathrm{G}} \cdot{ }^{17-19}$ We prepared seven FETs having different values of $V_{\text {th }}$ by varying $W$ from 290 to $600 \mathrm{~nm}$ and $L_{\mathrm{G}}$ from 100 to $690 \mathrm{~nm}$. Measured transfer curves for individual FETs are shown in Fig. 1(c). The obtained $V_{\text {th }}$ ranged from +82 to $-180 \mathrm{mV}$, a range of $262 \mathrm{mV}$. The variation in currents came from the differences in $W$. To enable us to observe the SR, we applied a common weak pulse train $V_{\text {in }}(t)$ to the gates as an input signal. The pulse height was as low as $14 \mathrm{mV}$, and the frequency and duty ratio were $100 \mathrm{~Hz}$ and $20 \%$, respectively. Each gate was biased with a dc offset $V_{\text {offset }}$ and uncorrelated white voltage noise was also added to each gate. The noise bandwidth was $50 \mathrm{kHz}$, which was sufficiently larger than the input signal frequency. Noise was measured as the root mean square (rms) of the voltage noise $V_{\text {noise }} . I_{\text {out }}$ was obtained by measuring $I_{\mathrm{DS}}$ in each FET individually and summing the values on a computer. The system response was

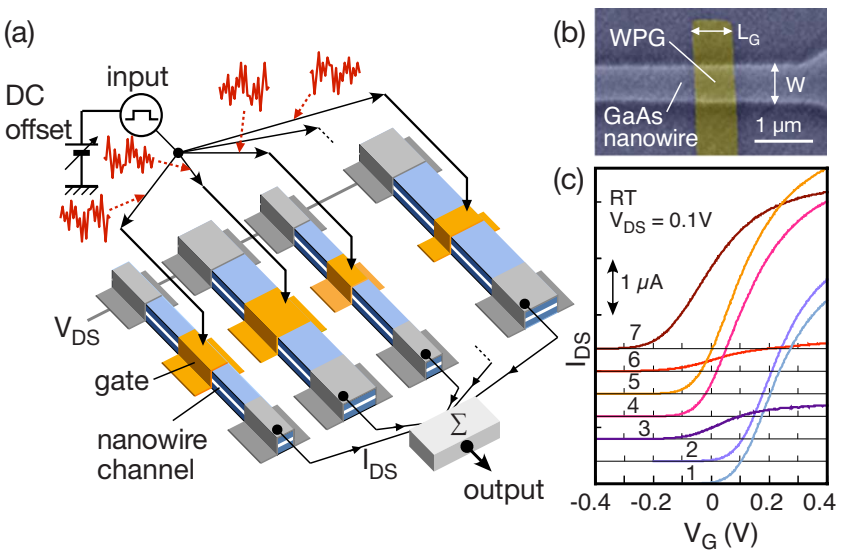

FIG. 1. (Color online) (a) Schematic of nanowire FET summing network with $V_{\text {th }}$ variation. The nanowire width and gate length were artificially modulated to achieve $V_{\text {th }}$ variation. (b) Scanning electron micrograph of a fabricated FET. (c) Measured transfer characteristics of seven FETs in the network. 


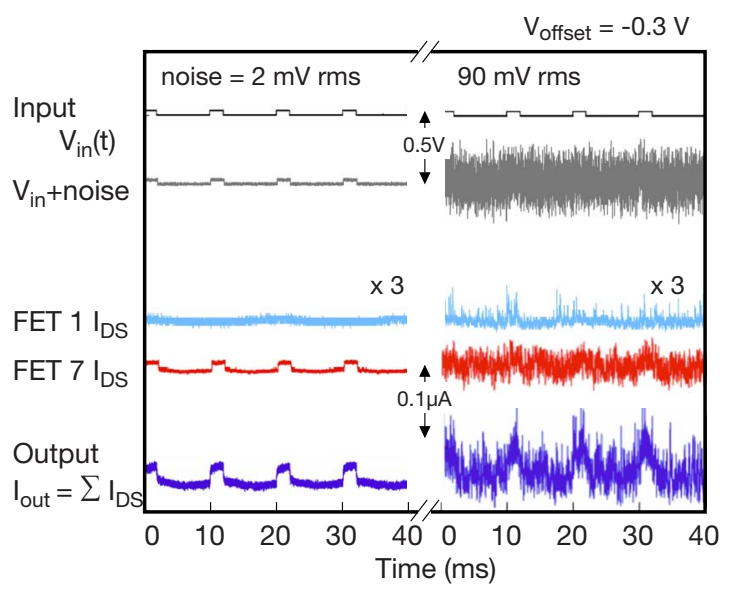

FIG. 2. (Color online) Measured waveforms of the varied FET network when low and high noise was added at $V_{\text {offset }}=-0.3 \mathrm{~V}$. Outputs of FETs 1 and 7 in Fig. 1(a) are also shown.

evaluated in terms of the correlation coefficient $C_{1}$ between input and output waveforms defined by

$$
C_{1}=\frac{\left\langle V_{\text {in }} I_{\text {out }}\right\rangle-\left\langle V_{\text {in }}\right\rangle\left\langle I_{\text {out }}\right\rangle}{\sqrt{\left\langle V_{\text {in }}^{2}\right\rangle-\left\langle V_{\text {in }}\right\rangle^{2}} \sqrt{\left\langle I_{\text {out }}^{2}\right\rangle-\left\langle I_{\text {out }}\right\rangle^{2}}},
$$

where $\left\langle V_{\text {in }}\right\rangle$ denotes the ensemble average of $V_{\text {in }}(t)$. It is important to note that this equation gives a meaningful information only when there is no output phase shift. ${ }^{14,20}$ In this study, the input signal frequency was set much lower than the FET cut-off frequency and the noise bandwidth, thus the phase shift could be ignored and we used Eq. (1). In the case with the phase shift, the cross-spectra method is better for evaluation. ${ }^{14,20}$ All measurements were carried out at room temperature. Extrinsic noise in the measurement system was $2.5 \mathrm{mV}$ rms, which was small and did not affect the measurement.

Examples of measured waveforms in the varied FET network when low and high noise was added are shown in Fig. 2. Outputs from two FETs, corresponding to 1 and 7 in Fig. 1(c), are also shown. The responses obtained for individual FETs followed the theory; namely, the response depended on the distance between the input offset and the threshold voltage $\Delta V=V_{\text {offset }}-V_{\text {th. }}{ }^{15}$ The high- $V_{\text {th }}$ FET (labeled 1) showed no response when the noise was low but it began to respond as the noise increased. The low- $V_{\text {th }}$ FET (labeled 7) responded to the signal when the noise was rather low. The response degraded as the noise increased and the signal almost disappeared in the output when high noise was added. On the other hand, the behavior of the network was different from the above. The waveforms output from the network obviously showed a pulse train correlated with the input signal regardless of noise intensity. The response observed in the case of high noise was the typical SR of the summing network. A notable feature here was that the present system could respond to the input even when the noise was low.

Figure 3 shows measured input-output correlation coefficients as a function of noise voltage in a 7-FET network with uniform $V_{\text {th }}$ of $+80 \mathrm{mV}$ and with the varied $V_{\text {th }}$ in Fig. 1(c). $V_{\text {offset }}$ was changed from -0.4 to $+0.1 \mathrm{~V}$ with a step of $0.1 \mathrm{~V}$. An obvious discrepancy was found between the responses of the two systems. As shown in Fig. 3(a), the uniform FET network exhibited peaked curves when $V_{\text {offset }}$ $\leq-0.2 \mathrm{~V}$. The peak was higher and broader than that for the (a)

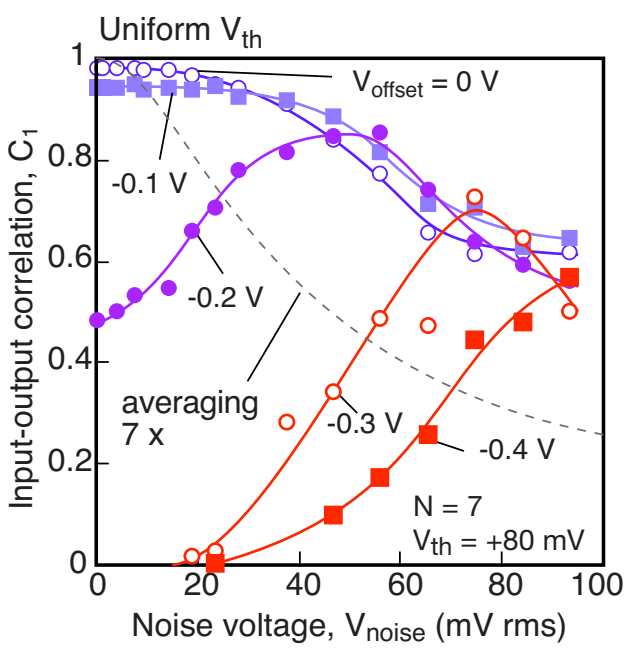

(b)

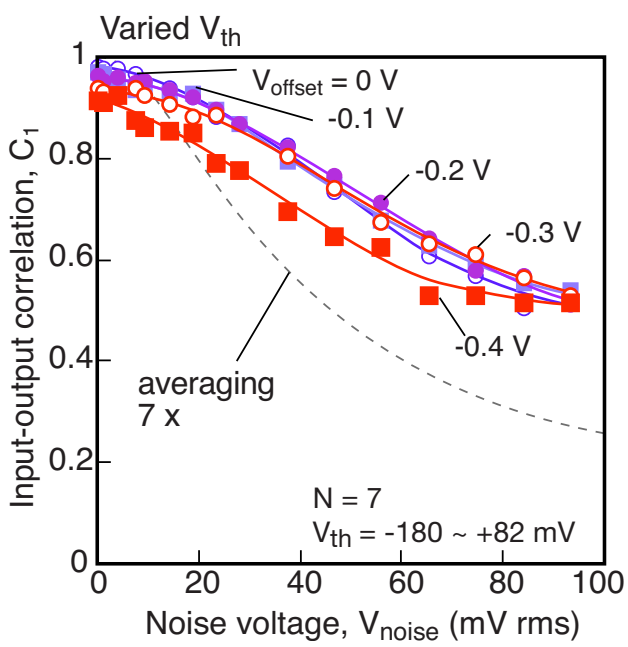

FIG. 3. (Color online) Input-output correlation coefficient as a function of noise voltage in the 7-FET summing network with (a) uniform $V_{\text {th }}$ and (b) varied $V_{\text {th }}$ for various values of $V_{\text {offset }}$. Broken lines indicate the response of a linear system after $7 \times$ sampling and averaging.

single FET (not shown). These were typical responses of the SR in the summing network. ${ }^{16}$ When $V_{\text {offset }}$ was increased, the peak position shifted toward the low noise side. Such behavior was the same as that of the single FET and it could be explained by the theory. ${ }^{15}$ When $V_{\text {offset }} \geq-0.1 \mathrm{~V}$, the network showed monotonically decreasing curves. This was because the distance between the threshold and the input offset was short and the peak position was close to $V_{\text {noise }}=0 \mathrm{~V}$. However, the correlation coefficient value was still larger than that of the linear system with seven-times sampling and averaging, plotted by dashed curves in Fig. 3. The slight decrease in the high-noise region at $V_{\text {offset }}=0 \mathrm{~V}$ was due to the weakened nonlinearity of the FET transfer curve, and the response gradually changed to that of the linear system.

The observed response of the varied FET network in Fig. 3(a) was different from that for usual SR. It hardly depended on $V_{\text {offset }}$ within the range of $V_{\text {th }}$ variation, indicating that the system adapted to the input offset fluctuation. The curve decreased monotonically with increasing noise, and no peak was seen. However, the slope was obviously gentle and the input-output correlation remained high compared with the linear system. These characteristics resulted in increased adaptability to the noise intensity fluctuation in the varied FET network compared with the uniform one. The response curves were similar to those for the uniform FET network in 


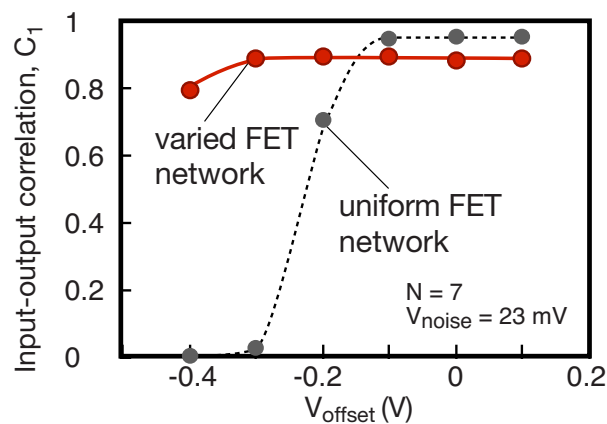

(a)

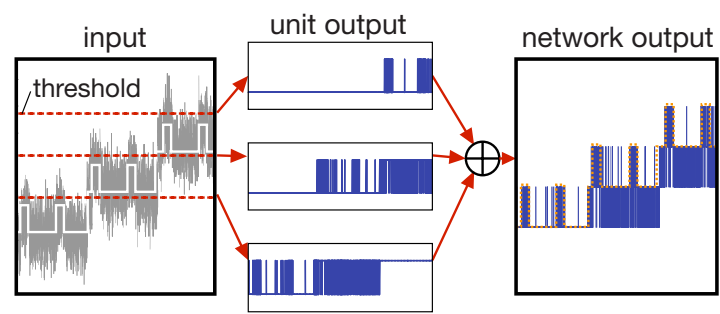

(b)

FIG. 4. (Color online) (a) Input-output correlation coefficients as a function of input offset voltage in varied and uniform $V_{\text {th }}$ FET summing networks and (b) the mechanism for increased dynamic range of the varied threshold system.

the case of $V_{\text {offset }} \geq-0.1 \mathrm{~V}$. It looked like that the distance between the threshold and the input offset voltages in the present system was always short, even when the offset was shifted.

Correlation coefficients of the uniform and varied FET networks with low noise of $V_{\text {noise }}=23 \mathrm{mV}$ are compared in Fig. 4(a). The varied FET network maintained a high correlation in a wider $V_{\text {offset }}$ range than the uniform FET network. This behavior was similar to that obtained in the uniform network when moderate noise was added. ${ }^{16}$ This result clarifies that noise in the signal and variation in the threshold contribute similarly to SR. The observed adaptability to the input offset fluctuation and increased sensitivity with low noise in the varied FET network can be explained by an enhancement of the dynamic range due to multiple thresholds and a summation process. ${ }^{21}$ The basic concept is schematically shown in Fig. 4(a). In this system, an input signal distributed over a wide range is decomposed by several units having different thresholds. In the case of a step function with a different threshold for each unit, one unit can detect only part of the input owing to the insufficient dynamic range, as shown in the middle of Fig. 4(b). However, different units separately detect different parts of the input. After that, the input signal is reconstructed simply by summing the decomposed signals, as shown on the right of Fig. 4(b). We found that the varied-threshold network allows the input to cross one of the thresholds when low noise is added. This results in sensitive signal detection in the case of low noise. In addition, the response of the summing network is domi- nated by the highest unit among all the units. Computing the correlation coefficient in a summing network consisting of linear units for simplicity, we obtain

$$
C_{1}=\frac{1}{\sqrt{1+\frac{N}{\left(\sum_{i=1}^{N} C_{1 i} / \sqrt{1-C_{1 i}^{2}}\right)^{2}}}},
$$

where $N$ is the number of units and $C_{1 i}$ is the correlation coefficient of the $i$ th unit. This formula indicates that the largest $C_{1 i}$ in the summing network dominates $C_{1}$ of the total system. The above two mechanisms enable the network to achieve high input-output correlation for a wide range of offset and noise intensity fluctuations.

In conclusion, SR in a summing network with varied thresholds was investigated using GaAs-based etched nanowire FETs. The system was found to detect a weak signal without any adjustment of the input offset or the addition of high noise. The improvement in adaptability was explained by a widening of the system's dynamic range. A wide range signal could be detected by decomposing multiple thresholds and reconstructed by summing them. The obtained results clarified that a summing network can exploit the threshold variation in network units to detect a weak signal in the presence of noise.

${ }^{1}$ T. Oya, T. Asai, and Y. Amemiya, Chaos, Solitons Fractals 32, 855 (2007).

${ }^{2}$ A. Utagawa, T. Sahashi, T. Asai, and Y. Amemiya, IEICE Trans. Fundamentals E92-A, 2508 (2009).

${ }^{3}$ F. Hartmann, D. Hartmann, P. Kowalzik, L. Gammaitoni, A. Forchel, and L. Worschech, Appl. Phys. Lett. 96, 082108 (2010).

${ }^{4}$ K. Nishiguchi, S. Miyamoto, and A. Fujiwara, 22nd International Microprocesses and Nanotechnology Conference, Sapporo, Japan, 16-19 November 2009 (unpublished), digest of papers, pp. 470-471.

${ }^{5}$ R. Benzi, S. Sutera, and A. Vulpiani, J. Phys. A 14, L453 (1981).

${ }^{6}$ L. Gammaitoni, P. Hanggi, P. Jung, and F. Marchesoni, Rev. Mod. Phys. 70, 223 (1998).

${ }^{7}$ T. Shimozawa, J. Murakami, and T. Kumagai, in Sensors and Sensing in Biology and Engineering, edited by F. G. Barth, J. A. C. Humphrey, and T. W. Secomb (Springer, Berlin, 2003), pp. 145-157.

${ }^{8}$ S. Fauve and F. Heslot, Phys. Lett. A 97, 5 (1983).

${ }^{9}$ A. D. Hibbs, A. L. Singsaas, E. W. Jacobs, A. R. Bulsara, J. J. Bekkedahl, and F. Moss, J. Appl. Phys. 77, 2582 (1995).

${ }^{10}$ X. Godivier, J. Rojas-Varela, and F. C. Blondeau, Electron. Lett. 33, 1666 (1997).

${ }^{11}$ R. N. Mantegna and B. Spagnolo, Phys. Rev. E 49, R1792 (1994).

${ }^{12}$ I. Lee, X. Liu, C. Zhou, and B. Kosko, IEEE Trans. NanoTechnol. 5, 613 (2006).

${ }^{13}$ K. Loerincz, A. Gingl, and L. B. Kiss, Phys. Lett. A 224, 63 (1996).

${ }^{14}$ L. B. Kiss, in Chaotic, Fractal, and Nonlinear Signal Processing, edited by R. Katz (AIP, New York, 1996), pp. 382-396.

${ }^{15}$ S. Kasai and T. Asai, Appl. Phys. Express 1, 083001 (2008).

${ }^{16}$ J. J. Collins, C. C. Chow, and T. T. Imhoff, Nature (London) 376, 236 (1995).

${ }^{17}$ M. Yumoto, S. Kasai, and H. Hasegawa, Appl. Surf. Sci. 190, 242 (2002).

${ }^{18}$ S. Kasai, T. Nakamura, and Y. Shiratori, Appl. Phys. Lett. 90, 203504 (2007)

${ }^{19}$ Y. Shiratori and S. Kasai, Jpn. J. Appl. Phys. 47, 3086 (2008).

${ }^{20}$ S. Sethuraman and L. B. Kish, Proc. SPIE 5110, 244 (2003).

${ }^{21}$ L. Gammaitoni, Phys. Lett. A 208, 315 (1995). 